\title{
Renal Subcapsular Hematoma after Intravenous Thrombolysis in a Patient with Acute Cerebral Infarction
}

\author{
Yun Kyung La, MD', Ji Hwa Kim, MD', Kyung-Yul Lee, MD, PhD', 2
}

A 74-year-old female with acute cerebral infarction was treated with intravenous recombinant tissue plasminogen activator. Subsequent percutaneous transfemoral angiography and mechanical thrombectomy were performed due to a right middle cerebral artery occlusion, which was successfully recanalized. Two days after treatment, the patient complained of vague right abdominal pain and a laboratory test showed anemia. Abdominal computed tomography showed a right renal subcapsular hematoma. After conservative management, the patient was discharged without complications. We report a rare complication after intravenous thrombolysis in a patient with acute cerebral infarction.

Key Words : Renal subcapsular hematoma; Intravenous thrombolysis;

Percutaneous transfemoral angiography

Renal subcapsular hematoma is a rare form of intraabdominal bleeding. Subcapsular hematomas can be spontaneous or iatrogenic, and iatrogenic causes include renal biopsy, anticoagulation, extracorporeal shock wave lithotripsy, hemodialysis and renal transplantation [1]. Intravenous recombinant tissue plasminogen activator (rTPA) is used in acute cerebral infarction to recanalize an occluded vessel. However,

Departments of ${ }^{1}$ Neurology, Gangnam Severance Hospital and ${ }^{2}$ Severance Institute for Vascular and Metabolic Research, Yonsei University College of Medicine, Seoul, Korea

Received August 24, 2016; accepted after revision August 26, 2016.

Correspondence to: Kyung-Yul Lee, MD, PhD

Department of Neurology, Gangnam Severance Hospital and Severance Institute for Vascular and Metabolic Research, Yonsei University College of Medicine, 211 Eonju-ro, Gangnam-gu, Seoul 06273, Korea.

Tel. 82.2.2019.3325 Fax. 82.2.3462.5904

E-mail: kylee@yuhs.ac.

This is an Open Access article distributed under the terms of the Creative Commons Attribution Non-Commercial License (http://creativecommons.org/licenses/by-nc/3.0) which permits unrestricted non-commercial use, distribution, and reproduction in any medium, provided the original work is properly cited. intravenous rTPA can cause bleeding complications, such as intracerebral hemorrhage, gum bleeding, gastrointestinal bleeding, and oozing from intravenous catheter sites. We present a rare case of renal subcapsular hematoma after intravenous rTPA and subsequent percutaneous transfemoral angiography.

\section{CASE PRESENTATION}

A 74-year-old female patient was admitted to our hospital with acute left-sided weakness. She had a history of hypertension and myocardial infarction with coronary artery stent insertion. She had been taking $100 \mathrm{mg}$ aspirin per day since coronary artery stenting. Her initial vital signs yielded a blood pressure of $107 / 60$ and heart rate of 81 beats per minute.

Initial neurologic examination showed eyeball deviation to the right side, left-sided hemiplegia and visual extinction, and the National Institutes of Health Stroke Scale (NIHSS) score was 13. Brain computed tomography (CT) angiography showed occlusion of the right middle cerebral artery (MCA). Intravenous rTPA 
infusion was started 3 hours after symptom onset and a subsequent percutaneous cerebral angiogram was performed via right femoral artery access. A 5-Fr Revive IC catheter was introduced into the right internal carotid artery and mechanical thrombectomy with a Solitaire retriever was attempted on the occluded right MCA, which was successfully recanalized (Fig. 1A, B). Follow-up brain MRI showed acute cerebral infarction in the right MCA territory (Fig. 1C). Because there was severe hypokinesia of the left ventricle on transthoracic echocardiography, we planned to use anticoagulation to prevent stroke recurrence.
Two days after the thrombolysis, the patient complained of vague right abdominal pain, and a laboratory test showed anemia. The patient's hemoglobin initially dropped to $9.5 \mathrm{~g} / \mathrm{dL}$ from $13.1 \mathrm{~g} / \mathrm{dL}$. We did not observe oozing from the puncture site, abdominal or posterior pelvic wall hematoma or signs of gastrointestinal bleeding such as melena, hematochezia or hematemesis. We performed contrast-enhanced abdominal $\mathrm{CT}$ and found a right renal subcapsular hematoma with mild compression of the renal parenchyma (Fig. 2). We delayed anticoagulation due to the renal hematoma and started anticoagulation 12 days
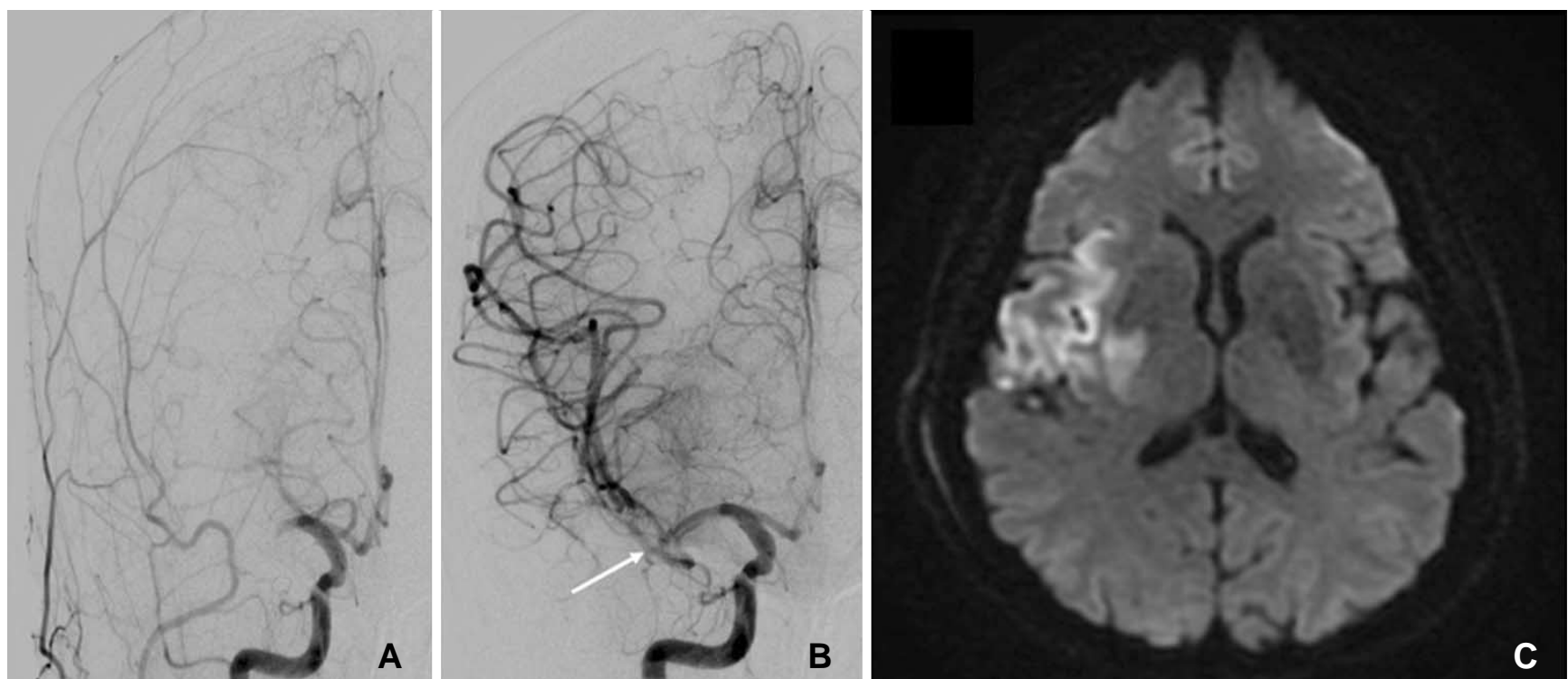

Fig. 1. Cerebral angiography shows occlusion of the right middle cerebral artery $(\mathbf{A})$ and successful recanalization after mechanical thrombectomy with a Solitaire retriever (arrows) (B). Brain MRI demonstrates acute infarction of the right basal ganglia and right middle cerebral artery territory $(\mathbf{C})$.
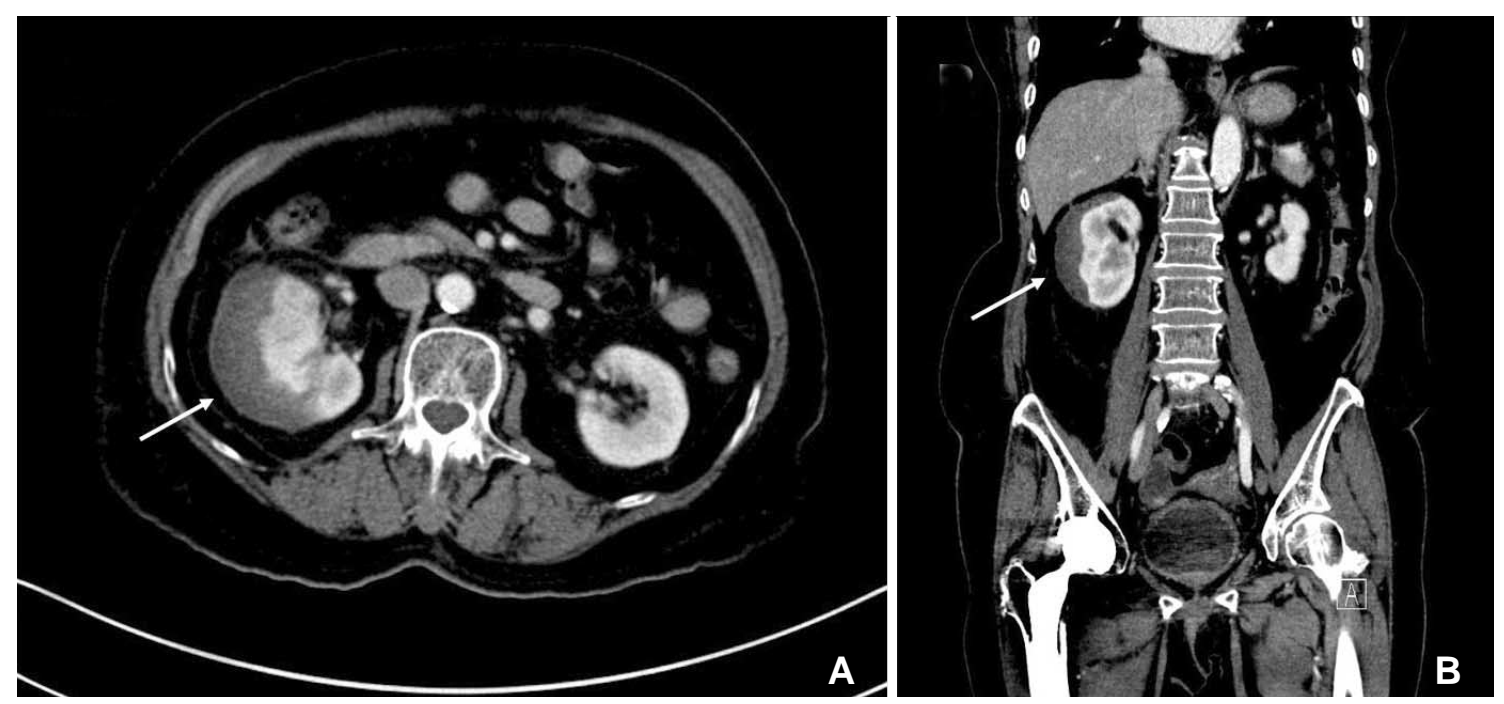

Fig. 2. Axial (A) and coronal (B) contrast-enhanced abdominal computed tomography (CT) reveal right renal subcapsular hematoma (arrows). 

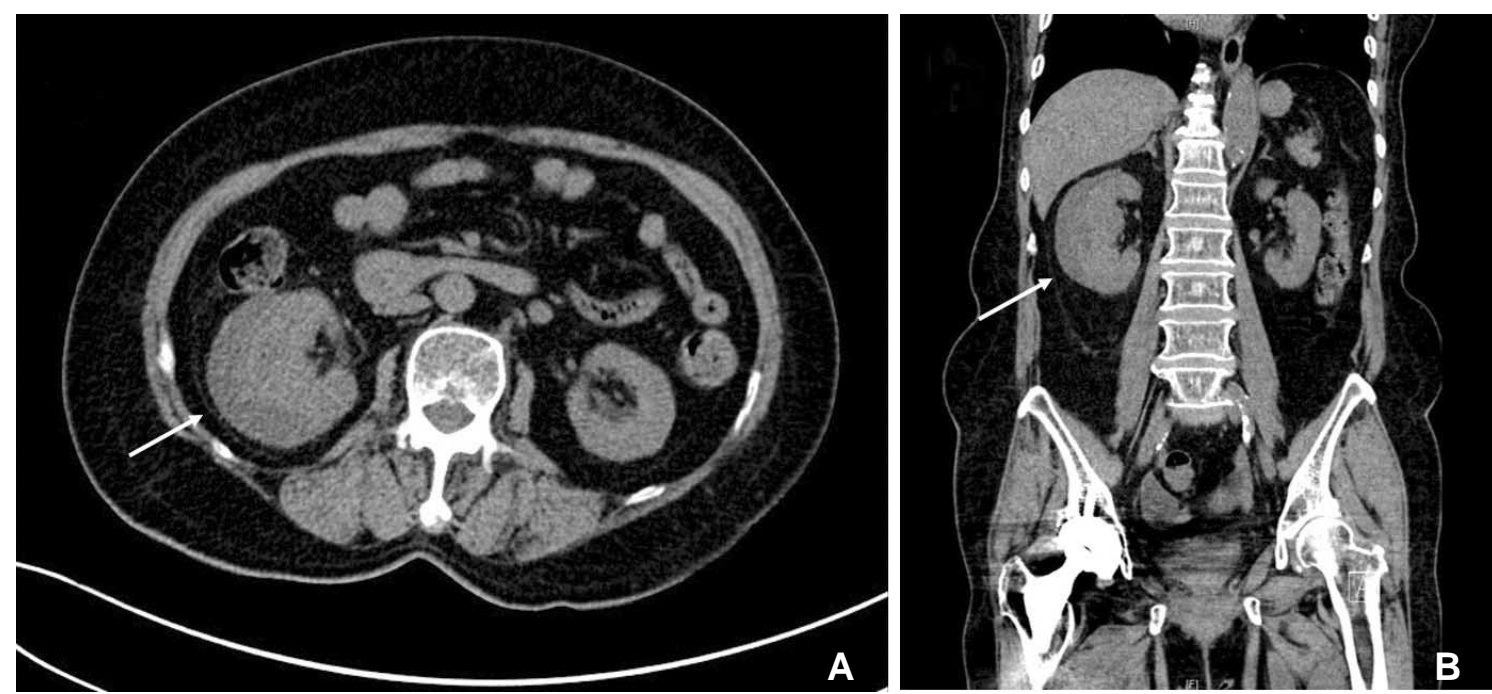

Fig. 3. Axial (A) and coronal (B) non-contrast abdominal CT performed 4 weeks after initial CT show decreased volume of the subcapsular hematoma of the right kidney (arrows).

after thrombolysis, after we had confirmed the lack of further hematoma growth on follow-up non-contrast abdominal CT. A follow-up non-contrast abdominal CT scan one month later showed regression of the hematoma. The patient's neurologic symptoms had also improved, with an NIHSS score of 1 (Fig. 3).

\section{DISCUSSION}

This case shows a rare complication of reperfusion therapy in acute cerebral infarction. There were two possible causes of renal subcapsular hematoma in this case: intravenous rTPA infusion and transfemoral cerebral angiography. Cerebral hemorrhage after intravenous rTPA use occurs commonly, and symptomatic intracranial hemorrhage has been reported in $6.8 \%$ of treated patients [2]. However, remote hemorrhage outside the brain occurs rarely, and there have been no previous reports of renal subcapsular hematomas [3].

Diagnostic angiography with transfemoral catheterization can have vascular complications, with an incidence rate estimated to be $0.3-1 \%$. These complications include localized arterial fistula or aneurysm at the puncture site, arterial occlusion, atheroembolism of the distal part, stent migration, renal artery dissection, and aortic dissection $[4,5]$. Hematomas are usually located in the femoral puncture site, retroperitoneal space, intraperitoneal region, and abdominal wall; however, renal subcapsular hematomas are very rare $[6$, 7].

There have been only three case reports describing renal subcapsular hematoma after transfemoral angiography $[1,8,9]$. In these cases, patients complained of abdominal or flank pain immediately after the procedure. In our case, the angiography procedure was done without any difficulty and abdominal pain developed two days after the procedure, which was late compared to the reported cases of renal subcapsular hematoma. Therefore, we speculated that the renal subcapsular hematoma was caused by intravenous rTPA infusion and that this type of complication had not been previously reported. Since antithrombotic or anticoagulation medication could worsen the renal subcapsular hematoma, clinicians should consider this type of complication in acute ischemic stroke patients with abdominal pain after thrombolysis. As a history of hypertension cannot be completely ruled out of aggravating spontaneous renal subcapsular hematoma, strictly controlling of blood pressure is also important [10]. This case highlights that careful medical observation is needed in patients with acute cerebral infarction who undergo reperfusion therapy.

\section{References}

1. Yi JS, Lee HJ, Lee HJ, Yang JH. Renal Subcapsular Hematoma after Percutaneous Transfemoral Angiography. J Korean Neurosurg Soc 2014;55:96-98

2. Emberson J, Lees KR, Lyden P, Blackwell L, Albers G, Bluhmki E, et al. Effect of treatment delay, age, and stroke severity on the effects of intravenous thrombolysis with alteplase for acute ischaemic stroke: a meta-analysis of individual patient data from randomised trials. Lancet 2014;384:1929-1935

3. Romano JG, Smith EE, Liang L, Gardener H, Camp S, Shuey L, 


\section{Yun Kyung La, et al.}

et al. Outcomes in mild acute ischemic stroke treated with intravenous thrombolysis: a retrospective analysis of the Get With the Guidelines-Stroke Registry. JAMA Neurol 2015;72:423-431

4. Johnson L, Lozner E, Johnson S et al. Registry Committee of the Society for Cardiac Angiography (1989) Complications of cardiac catheterization: Coronary arteriography 1984-1987: A report of the Society for Cardiac Angiography and Interventions. I. Results and complications. Cathet Cardiovasc Diagn 1878;17:5-10

5. Ricci MA, Trevisani GT, Pilcher DB. Vascular complications of cardiac catheterization. Am J Surg 1994;167:375-378

6. Xia D, Chen SW, Zhang HK, Wang S. Renal subcapsular haematoma: an unusual complication of renal artery stenting. Chin Med J (Engl) 2011;124:1438-40

7. Perkovic V, Thomson KR, Mitchell PJ, Gibson RN, Atkinson N,
Field PL, et al. Treatment of renovascular disease with percutaneous stent insertion: long-term outcomes. Australas Radiol 2001;45:438-443

8. Fang CC, Ng Jao YT, Han SC, Wang SP. Renal subcapsular hematoma after cardiac catheterization. Int J Cardiol 2007;117: e101-103

9. Kwon SH, Cho HC, Lee SW, Kim DY, Joo WC, Lee WH, et al. A case of right renal infarction and subcapsular hematoma that simultaneously developed after cardiac angiography. Clin Nephrol 2009;71:84-87

10. Baishya RK, Dhawan DR, Sabnis RB, Desai MR. Spontaneous subcapsular renal hematoma: a case report and review of literature. Urol Ann 2011:3:44-46 